Pankoke-Babatz, U. \& Jeffrey, P. "Documented Norms and Conventions on the Internet". (June 2002). In Luczak, H. International Journal of Human-Computer Interaction (Special Issue on Awareness),14(2), Lawrence Erlbaum Association,219-235.

\title{
Documented Norms and Conventions on the Internet
}

\author{
Uta Pankoke-Babatz and Phillip Jeffrey \\ GMD - German National Research Center for Information Technology \\ Schloß Birlinghoven \\ D-53754 Sankt Augustin, Germany \\ uta.pankoke@gmd.de,phillipj@cs-club.org
}

\begin{abstract}
The Internet plays an important role as a means for worldwide social contact. The establishment and maintenance of social and group relationships within electronic worlds require social norms and behavioural conventions as in the real world. This paper investigates some of the available electronic media: Email, mailinglists, newsgroups, chatrooms, and MUDs. Peculiarities of the media are analysed through studying the documented behavioural norms and social conventions. We have looked at desired behaviour, disruptive behaviour and at sanction mechanisms. A conclusion that may be drawn is that within these virtual environments, explicitly documented norms and conventions play an underlying role in how individuals behave in addition to the type of behaviour that is expected from others.
\end{abstract}

\section{Introduction}

The Internet, similar to previous inventions (i.e. television, radio, and telephone), has had a dual purpose as traditional barriers of physical distance separating societies have not only disintegrated, but individuals, groups, communities, and nations are now part of the 
interconnectivity of a networked environment. A community no longer is perceived within physical dimensions or limited by physical boundaries. The cultural norms, values and ideas that shape members of that community are linked and shared with other communities through virtual environments such as chatrooms, MUDs (Multi-user Dungeons or Multi-user Domains) and newsgroups. Within a shared virtual environment, individuals and groups collaborate, interact, and communicate in one space that through shared meaning, understanding, and identification may be perceived as a place.

The use of the Internet is no longer restricted to a small group of technically engaged people, instead it is becoming a common means for ordinary people to establish and maintain social relationships. This requires individuals to develop an understanding of how social relations may be established across time and space, in which people are connected through their virtual rather than physical presence. In real world face-to-face settings, an important mechanism to achieving congruent behaviour is provided through the co-presence of observing others and adjusting one's behaviour accordingly. However in most current electronic environments, there is a lack of mutual awareness and a low level of social presence (Short et al., 1976). The lack of implicit means to achieve congruent behaviour requires that explicit means be used to develop and communicate behavioural rules. Aspects of these virtual environments that may mirror the physical world include the establishment and application of behavioural rules or social conventions that regulate behaviour. Understanding the existing rules provided for social interaction may enhance one's understanding of social behaviour on the Internet.

The aim of this paper is to develop a better understanding of these behavioural rules and social conventions and how they are being applied on the Internet. We begin with a brief outline of the role that norms and conventions play within the real world and their 
relationship to the environment. Next, we will outline the role of the Internet in the community building process. We present the most relevant asynchronous (Email, newsgroups, mailinglists) and synchronous communication media (chatrooms, MUDs) based on the documentation provided in the literature. For each medium we will analyse a selection of rule sets currently documented as netiquettes on the Internet. We will learn how netiquettes relate to real world conventions, how they aim to support desired behaviour, and attempt to contribute to the prevention or sanction of undesired behaviour. Particular attention will be paid to how netiquettes propose to cope with the low level of social awareness and social presence provided within these media. From this analysis we will learn about the management of social relationships within electronic media. We conclude with a comparison of the different media based on what was learned from the written netiquettes.

\section{Background of the Work}

Social norms or moral rules play an essential role in our existence within society. Following these rules helps ensure one's survival, happiness, and well being as a societal member. Rules exist not only for the benefit of an individual but extend to offer a collective benefit. This ensures that order exists within the culture so that individuals of different backgrounds and different facets of thinking can function together by knowing and following societal rules. Communication, common goals, and the high opportunity for contact facilitate the group forming process (Crott, 1979, p. 217). Therefore the Internet creates new possibilities for contacting people throughout the world, as well as providing opportunities for groups to form. 


\subsection{The Role of Social Norms}

Group forming processes are guided by the principles of proximity, similarity, reinforcement, symmetry, and co-orientation, in addition to affect and emotions (Davis \& Palladino, 1995). An essential part of the group forming process is the establishment of social norms and behavioural conventions, whether explicitly defined as rules or laws, or implicitly defined through acknowledged or sanctioned behaviour. Group norms "develop through explicit statements by supervisors or co-workers, critical events in the group's history, primacy, or carry-over behaviour from past situations". Norms should help "ensure group survival and increase the predictability of group members' behaviour and avoid embarrassing interpersonal situations" (Feldman, 1984, p. 47-51).

According to Mann (1972, p. 64) a large part of in-group communication refers to the maintenance of conformity. In addition, there is a close relationship between conformity of group members and group cohesiveness (Davis and Palladino, 1995, p. 683). Knowing and applying the right norms and conventions gives each participant the feeling of being part of the group. It may then also contribute to an easier achievement towards a common understanding and cooperation among group members.

In electronic group forming processes, group rules encoded in norms and conventions may even be more important for a number of reasons: as a means to develop shared attitudes, to achieve congruent reactions, to contribute to achieving a mutual understanding, and to create a social reality. We expect that the lack of awareness and the low level of social presence in these media will be reflected in the behavioural rules.

\subsection{The Role of Places}

In the group forming and maintenance process, environments play an important role as places where people meet and interact. Barker and Wright (1955) have concluded that 
individual behaviour also depends on the affordances of a given environment. They found a structural similarity between individual behaviour in the form of standardised behaviour patterns and environments in the form of physical and social milieu. Environments and artefacts influence the standing pattern of behaviour through their physical properties and social connotations. Milieu-conforming behaviour is learned by trying something out, observing the impact, watching others, and through imitation.

Oldenburg (1989) has been studying the societal importance of particular environments. He outlines how a common thread linking great civilisations and societies are informal public places such as a Biergarten in Munich or a sidewalk café in Paris, which are a vital locale for socialisation and relaxation. He notes that without such meeting places, inhabitants would be deprived of the types of relationships and human interaction, which are the lifeblood of every urban centre. These meeting places, which Oldenburg (1989, p16) labels as "third places" are fundamental to one's daily life as one's place of residence, which he called a "first place", and place of employment, considered a "second place". After the Industrial Revolution, the first and the second place separated.

\subsection{Places in the Internet}

A new type of immersion between places is occurring again involving this third place as individuals congregate online - in virtual communities. The Internet is recognised as an environment that supports forming communities (Reid, 1991; Herrmann, 1995; Turkle, 1996), so that a new way of community building is occurring as people are gathering through shared ideas rather than only through physical places. Virtual communities are described by Rheingold (1996, p. 418), as providing companionship for societal members deprived of human interaction, and as the global response to filling the gap left by the disintegration of Oldenburg's third places. Newsgroups, chatrooms and MUDs are all means to assemble 
people who share a common interest, without the limitation of gathering in the same physical location. Apart from behavioural rules, new terms have been created for this emerging culture such as: "smileys" and other novel forms of textual, emotive expressions as well as new terms such as "flaming" and "spamming". The particularities of these electronic places will influence the behaviour of their visitors (Pankoke-Babatz, 2000).

\subsubsection{The Role of Netiquettes}

A major difference between real world and Internet communication may lie in the fact that the Internet provides for the opportunity of non-physical and anonymous social interactions. The anonymity provided by the Internet may then encourage people to act with lowered inhibitions and result in establishing contacts easier. Relationships may reach a high level of intimacy in a shorter period if very personal details are being discussed in a public forum such as a newsgroup. An extreme example is the tinysex and cybersex behaviour prevalent in certain chatrooms (Hamman, 1996; Turkle, 1996). On the other hand, a relaxation of inhibitions may also result in unsociable behaviour and create an environment on the Internet that reduces the authenticity of human relationships (Rheingold, 1993, p. 147). Kollock and Smith (1994) believe that it is important for participants to adhere to the local rules for behaviour. Internet etiquette or netiquettes are intended to explain to new users (sometimes referred to as "newbies"), how to behave (Mandel \& van der Leun, 1996).

On the Internet we can differentiate between synchronous and asynchronous media. Synchronous media, such as chatrooms and MUDs allow almost immediate interaction. Email and newsgroups, as asynchronous media, provide time-dispersed communication where temporal delays between sending, reading and replying are common. In the following we will briefly describe each of the asynchronous media types. 


\subsubsection{Email}

Email has been available for over 20 years (Tannenbaum, 1996), although it has increased in popularity in the last 5 years. Email is a medium for bilateral and direct communication between friends, colleagues, acquaintances, as well as strangers. The first sent Email message similar to real life (Feldman, 1984), provides a first impression of the other party and may influence the type of relationship that develops. Therefore, it is necessary to facilitate a successful initial contact and learn about the netiquettes beforehand rather than through trial and error. 'Learning by doing' is possible with other media, however with Email, feedback is not immediately available. Since the early 80 s netiquettes for Email have been recommended in a number of studies (Brotz, 1983; Pankoke-Babatz, 1984).

\subsubsection{Newsgroups and Mailinglists}

Usenet, which began in 1979, organises worldwide discussions under a set of broad headings called "Newsgroups" (Rheingold, 1993, p. 69). A news-reading program presents those discussions in an orderly way. Newsgroups collect the comments of people around the world, in a way that enables people to address previous comments and thus, when functioning correctly, develop a kind of conversation that is documented in a "thread". A number of subcultures have developed in newsgroups. However, some newsgroups may be more similar in their behaviour to a battlefield rather than a community, although these will also have their regular users and social norms (Rheingold, 1993, p. 122).

Email based mailinglists enable individuals with common interests to collectively receive and sometimes individually 'post' (i.e. send information) to the distribution list (Kehoe, 1992). Mailinglists can be created by anyone, as the only requirement for membership is an

active Email address. In this paper, we have not treated mailinglists separately from newsgroups. 


\subsubsection{IRC and Chatrooms}

IRC stands for Internet Relay Chat. It is a popular, virtual environment where people around the world communicate online with each other using text and through thousands of channels based on a variety of different topics (Bechar-Israeli, 1995). IRC technology was developed in 1988 by Jarkko Oikarinen at the University of Oulu in Finland (Hamman, 1997). The purpose of IRC is social communication (Kelley, 1995) in real time. Once a user logs onto an IRC server, he or she joins one of the channels of interests and engages in conversation.

Chatrooms are multi-user environments that provide textual communication with others in real time. Anyone can interact in a chatroom. Two examples are the travel guidebook in the Let's Go chatroom (Letsgo, 1997) and the more multi-purpose WBS environment (WBS, 1998).

\subsection{MUDs}

MUDs were created around 1978 by Roy Trubshaw for the purpose of designing a multiplayer computer adventure game (Hamman, 1996) and were originally called "Multi-user Dungeons". Today the name "Multi-user Domains" is more common. From 1990 onwards their number and usage has increased as MUDs for social interactions have become more prevalent (Reid, 1994).

Textual descriptions provide a metaphor of a physical environment. As well, each MUD has build-in rules that outline the types of interactions that can occur and how the culture within the world should develop (Mitchell, 1996, p. 119). Before joining, a player must create a character that usually involves selecting a name, gender, and providing a description. This character is the person's representation through which actions and communication occur. As the real-world gender of a player cannot be confirmed online, the opportunity exists for experimentation with gender-swapping (Reid, 1996; Turkle, 1996). In contrast to 
the other media described, interaction is not limited to exchanging textual messages with other players but players can also express non-verbal utterances with their avatars such as smiling or gestures. Players may interact with the environment itself, thus modifying it, e.g. in some MUDs structures such as buildings can be created.

MUDs may be considered virtual environments. In purely text based MUDs, players imagine the virtual world, while in 3-D MUDs the environments are visualised. The objectoriented variant of a MUD is called MOO (Lavato, 1997; Morgenes, 1997; Lavato, 1998; Morgenes, 1997) and is a more recent development. The object orientation facilitates more complex and easier to use interaction modes to the players.

Although MUDs began as adventure games, they also exist today as social spaces (Bruckman \& Resnik, 1995) or as learning environments. LambdaMOO, created by Pavel Curtis (1996, p. 351) in 1990, is a well-known virtual environment with about 8000 participants, comparable to a small virtual city. Turkle (1995, p. 181) views MUDs as a hybrid activity between programming and "writing fiction".

\section{Evaluation of Netiquettes}

We use the term "netiquette" to refer to the documented behavioural norms and social conventions that we found on the Internet. With the growth of the Internet, the relevance of netiquettes is increasing. We conclude this from the high number of netiquettes found, as an Internet search request (using AltaVista) with the term "netiquette" led to more than 80000 references in Spring 2000.

The rule sets provide evidence about what is considered relevant in the particular communities, however they do not aim to be comprehensive. The analysis of the rule sets may help one understand media specific differences. In our analysis we pay particular attention to how people handle the minimal level of mutual awareness and social presence 
provided by these media. We expect considerable differences between synchronous and asynchronous media and we are interested to see how media specific differences might be reflected in the netiquettes.

\subsection{Methods}

We combine the social behavioural findings that are specific to media, as described above, with those documented in the sets of netiquettes. For this subsequent purpose, we collected rule sets on the Internet, between November 1999 and February 2000, using several search engines (BullsEye, AltaVista, and Yahoo) and by combining terms (Email \& conventions; newsgroups \& conventions...). We tabulated 18 general sets for Internet usage, 14 sets for Email, 24 for newsgroups and mailinglists, 18 for chatrooms and 13 for MUDs to provide a more detailed analysis.

Netiquettes for the Internet in general, varied between 10 rules on a single page (Netiquette, 1997) and 100 rules spread over 12 pages (RFC1855, 1995) when containing separate subsets for each medium. Email had a mean of 18 rules on 6 pages, newsgroups 13 rules on 5 pages, chatrooms 10 rules on 3 pages, and MUDs 17 rules on 5 pages.

$92 \%$ of the netiquettes for Email and $71 \%$ for newsgroups were available as separate rule sets. Personal websites for Email and general rules were used to provide relevant information as a service to the Internet community. For us this indicates that netiquettes for Email and newsgroups are more general and independent from particular instances of service. The conventions for chatrooms, and MUDs were more specific to the particular service and usually found at the entrance of that particular site $(72 \%$ of the netiquettes for chatrooms and 61\% for MUDs) (MOO Connections, 2000; About, 2000).

Through our detailed analysis of the rule sets, we differentiate between the following major aspects: the desired behaviour, the abusive (undesired) behaviour, and sanction 
mechanism available. For each of these aspects we defined a set of categories that were used for encoding the rule sets. One author performed the initial encoding; the other author checked the encoded sets.

\subsection{Desired Behaviour in Netiquettes}

Netiquettes attempt to give optimal benefit of the medium to its users and to achieve an even distribution of effort and benefit. We have differentiated the rules for desired behaviour into the following categories: awareness of audience, privacy, politeness, style, and roleplaying.

\section{Table 1: Percentage of netiquettes addressing desired behaviour}

\subsubsection{Application of Real World Rules}

Cooperation within the Internet will only function when following social rules that have proven successful in the real world. Therefore some of the rules may be transferred from the physical world. References to real world etiquette appear in the netiquettes for each of the media. As the following two examples indicate, rules may be explicitly referenced. The first example is for the Internet in general, the second is taken from the entrance homepage of a chatroom:

"remember the human" and "adhere to the same standards of behavior online that you follow in real life" (Netiquette, 1997).

"...Your conduct should be guided by common sense, basic etiquette, and these chat rules" (America Online, 1999). 
A very brief and comprehensive set of general rules can be found in the netiquette (Netiquette, 1997) by Shea, which is one page containing ten rules. Many other netiquettes sites provide links to this particular set.

\subsubsection{Awareness of Audience and Privacy}

Particular to the media, $56 \%$ to $85 \%$ of the netiquettes contain rules that refer to the awareness of audience, although the presence of the audience may not be entirely perceived. Those rules remind the users of the fact that many people may be able to read what is written and be personally affected by it.

In the case of Email, rules that enhance awareness regarding lack of control about the future use of an Email message would fall under this category (i.e. once sent the recipient may actually forward it). This may be combined with the issue of privacy, as rules often address both the perspectives of the actor and the audience:

"Never send anything you would not want to see in tomorrow's newspaper" (Email Etiquette, 1999).

For newsgroups, rules of the category awareness of audience recommend that one orient oneself towards the particular aim and culture of the group. Although it is impossible to become aware of other participants' behaviour, documentation of past discussions may provide some indication of adequate behaviour. Netiquettes often recommend that one observe the ongoing discussion for a period of time (called lurking) before becoming an active participant. This enables one to learn from others about expected behaviour, the desired style and mode of debate, and topics for discussion. Similar rules involving observation before interaction were also noted in the MUD and chatroom netiquettes: 
"Read both mailing lists and newsgroups for one to two months before you post anything. This helps you to get an understanding of the culture of the group" (RFC1855, 1995).

"Lurk, Subscribe to the group and read it for a while to get a feel' of the group" (ParadisePlace, 1999).

For chatrooms and MUDs, where anonymity is more prevalent, privacy is of particular relevance. Since users may create their own personae, privacy rules that convey conventions about when it is acceptable to talk about "real life" vary between different communities (i.e. a role-playing medieval adventure MUD compared with an educational MOO for a university course). The disclosure of someone's real world identity is considered in many groups not only to be a violation of privacy, but also is seen as a more offensive act (see section 3.3).

\subsubsection{Politeness and Role Playing}

Politeness (see Table 1) is a major issue in nearly every set, i.e. in $92 \%$ of the general sets, and in more than $75 \%$ in the specific media, with the exception of the netiquettes for chatrooms found, only $33 \%$ explicitly address politeness. Common rules in the netiquettes refer to an outlined code of conduct (use proper English, use prose command, use emoticons, respect others, be fair, be polite, protect privacy and confidence). A part of politeness is understanding and contributing to the aim of the community. These are media and community specific. We found that rules are adapted and refined according to the specifics of the media and the aim of the group.

In newsgroups it is recommended, that individuals follow the group habits, which may differ between newsgroups (Rospach, 1996; YOYO, 1997; Bayer, 1998). Concerns exist that many users only 'lurk' or ask questions without providing answers back to the group (Kollock \& Smith, 1994). Newsgroup netiquettes recommend reading the FAQs of a 
particular newsgroup before asking a question. One advantage of this is that unnecessary repetition of debate should be minimised. In addition, received answers could then be grouped into an FAQ.

In chatrooms it is often advised to use greeting and farewell conventions such as 'hello' and 'goodbye', although it is not necessary to acknowledge every person present. It may also be obvious to regular users who the new people are by their behaviour. MUDs and chatrooms sometimes advise new participants to identify themselves as such so that they may then be given assistance as well as some leeway in following conventions.

For MUDs we had to create an additional category called "Role Behaviour" to cover conventions that were specific to that particular MUD. For example, adventure or gamingtype MUDs suggest reviewing the history and background of the particular setting before joining the game in order to understand what role-playing behaviour is expected. MUDs have specific rules addressing anonymity and the virtual personae of the players, such as respect role-playing, act appropriately in public and be courteous as in real life (Ciskowski \& Benedikt, 1995).

\subsubsection{Style}

The issue of style is addressed particularly in the netiquettes for Email (86\% of the Email netiquettes). One interpretation is that Email may be a portal opening up a channel to a worldwide audience. Beginners do not have the opportunity to see firsthand examples of proper behaviour regarding Email messages. Although Email is usually personalised, it is not synchronous so that one cannot receive immediate feedback. From a historical perspective, the Email community is the largest and oldest. This has provided the community with the ability to develop its own style of writing through the use of emoticons and sets of acronyms. 
A typical rule with respect to style is the usage of capital letters, which is understood universally online as SHOUTING. It is considered appropriate only when used sparsely.

\subsection{Coping with Undesired or Disruptive Behaviour}

The importance of the social dynamics as induced by the nature of the underlying technical system becomes more apparent through what is considered offensive behaviour and through prevention and sanction mechanisms for abusive behaviour. From studying the rule sets, we will comment on areas of disruptive behaviour and focus on spamming and flaming in particular.

The following table (Table 2) illustrates that the prevention of undesired and abusive behaviour plays an important role in each kind of media. It shows that $71 \%$ to $94 \%$ of the netiquettes studied contain rules related to undesired behaviour. However we found media specific differences with respect to the kind of undesired behaviour to be prevented.

Table 2: Percentage of netiquettes containing rules concerning undesired behaviour

\subsubsection{Undesired Behaviour}

A particularly high percentage of netiquettes found for chatrooms (61\%) and MUDs $(82 \%)$ contain rules addressing undesirable behaviour. As stated earlier, for chatrooms this finding is caused by the fact that many netiquettes for chatrooms often list what behaviour should not occur (i.e. the don'ts) in the community:

“You agree to not use the Service to: ... 'stalk' or otherwise harass another" (Yahoo, 2000).

The disclosure of personal or anonymous information is considered to be an offensive act in many chatrooms and MUDs: 
“... never give out personal information to strangers" (Excite, 2000).

"Respect that user's desire for anonymity" (RFC1855, 1995).

In MUDs, another type of unsociable behaviour often mentioned is player killing:

"The 'kill' command is off-limits. If you want to role-play a death, make sure that the person dying has agreed to do so!" (Chrystal Mush, 1998).

Power gaming is another offensive behaviour mentioned in netiquettes for chatrooms and MUDs. This occurs when a player takes over the personae of another player and forces this player to act as he or she wishes. This may be as simple as using the nickname of another person in a chatroom or as extreme as rewriting code so that another avatar acts on one's behalf (Dibbell, 1998):

"If you're caught trying to mislead people by using certain nicknames to appear as if messages are coming from people other than yourself, you will be banned" (About.com, 2000).

"Please abide by the following code of conduct that prohibits......using fake names or impersonating others..." (CNN.com, 1999).

In the netiquettes for chatrooms and MUDs, conduct related to sexual abuse is often mentioned:

"Do not transmit via Excite Chat any information...that is unlawful: harmful: threatening: abusive: harassing: defamatory: vulgar: obscene; or racially or ethnically hateful..." (Excite, 2000). 
"We discourage any of the following: ...Post or reference sexually explicit images or other offensive content..." (America Online, 1999).

The ability to change one's gender has opened new opportunities to explore the other gender (Reid, 1991). But this also opens opportunities for misuse. Sexual behaviour such as cybersex or virtual violence may also happen in MUDs (Reid, 1996) and in chatrooms (Hamman, 1997). In a MUD, a player may find a way to control actions of another player's character and may force it to have sex. Turkle (1996, p. 55) understand this as virtual rape. Sexual attacks are explicitly forbidden in many netiquettes.

“...Sexual abuse is possible on MUDs, too, but naturally this is illegal” (Deeper Trouble, 2000).

\subsubsection{Spamming}

Spamming means to post long and frequent messages or send large amounts of newsgroup posting to hundreds of newsgroups or Email addresses. One reason that spam is a problem is that Email and newsgroups enable one to easily distribute commercial material. Recipients do not have a chance to sort out the topical contributions among the spammed material. It may also cause technical problems such as memory overflow or increase the local downloading time. This could result in the destruction of well-functioning groups, so it is in the interest of the community to find ways to prevent its occurrence. The wasted time spent unnecessarily downloading and subsequently deleting spam is not irrelevant, especially if the Email provider charges per message.

“...if you send spam expect to lose your net access” (ParadisePlace, 1999).

Each contribution for chatrooms must be short -about one line the length of the text window-, such that one can see several contributions in the chat window, dependent on the 
message length. Spamming will cause the visible chat window to be filled with the spam, rather than with message exchanges. This will remove any possibility of communication as the following example mentions:

"Do not cause any chat room screen in Excite Chat to scroll faster than other users are able to type to it or any action to a similar disruptive effect" (Excite, 2000).

\subsubsection{Flaming}

Flaming is a public personal attack. A simple unfriendly, rude personal comment may intentionally or unintentionally start a flame war (Astrian.net, 2000) if send to a public group rather than made as a private comment. The debate may easily become heated in a tempo that has no real world equivalent. The most offensive dynamics of flame wars occur within asynchronous communities (Dery, 1994, p. 1). The need to prevent flaming is of particular relevance to asynchronous media as indicated by the high percentage of general and newsgroup netiquettes explicitly addressing flaming (see Table 2). The absence of a social corrective mechanism and the ability of the abusers to be anonymous may contribute to uncontrollable flaming. Flaming may also result in people leaving the particular group, thus disabling previously well-functioning groups. As well, it may not be possible to keep on topic in a group that is overrun with flaming:

"You should not send heated messages (we call these "flames") even if you are provoked. On the other hand, you shouldn't be surprised if you get flamed and it's prudent not to respond to flames" (RFC1855, 1995).

Although spamming and flaming may actually cause problems in any of the media, time dispersed communication media such as newsgroups are more vulnerable against these types of disruptive behaviour (Kollock \& Smith, 1994). Respective contributions are instantly 
distributed, and immediate intervention is not possible in this media. Thus spamming and flaming may escalate and destroy a newsgroup before intervention, if at all possible, occurs. To prevent disruptive behaviour 'moderated' newsgroups have been created. In these newsgroups, contributions are forwarded to the moderator who then decides whether to accept and forward the contribution to the newsgroup as a whole, or whether to reject it. This protects the group against flaming and spamming. However this also presents the mediator with a high degree of control, which may not be suitable for all groups.

\subsubsection{Sanction Mechanisms}

Since netiquettes cannot prevent misuse, sanctions mechanisms are required. The intent of sanctions is two-fold; to immediately curtail the disruptive behaviour and to provide a deterrent through example. Social sanctions, such as a verbal reprimand or being referred to the location of posted netiquettes, exist. Sanction mechanisms are mainly mentioned in posted netiquettes for chatrooms (61\%) and MUDs $(65 \%)$ and less often in those for newsgroups (21\%) and Email (7\%). This could be attributed to the fact that disruptive behaviour can be more easily detected and addressed in synchronous media. Therefore sanction mechanisms can be much more effective than in asynchronous media.

Technical means, which enable people to individually protect themselves against disruptive people, are available as so called kill or ignore mechanisms in newsgroups as well as in most chatrooms and MUDs. These techniques block messages sent from the offending individual. Many of the netiquettes explicitly recommend the usage of these protection tools. "If you find yourself being harassed...you can quickly discourage the activity by clicking on CONFIG and selecting IGNORE for that person...Do not use profanities, or abusive language. Chat monitors have the authority to instantly boot an abusive chatter without warning” (Rainforest, 2000). 
However, ignore mechanisms do not stop an offender. Stronger sanctions may be applied in chatrooms or MUDs, where the administrators may be permanently online. Administrators may e.g. ban a person permanently (WBS, 1998), thus excluding the offender from any further use of the system. In addition, administrators may be able to immediately delete offensive material (HyperChat, 2000). Depending on the particular virtual community, the administrators have a high degree of power to define the rules and to sanction unwanted behaviour (Reid, 1991).

More extensive sanction mechanisms exist in MUDs and MOOs that may be labelled as rude. For example, the administrator, as a deterrent, may attach a note describing the abusive behaviour to the abuser's personae or avatar (Reid, 1999). Thus the potential of power gaming may be used a means for an administrator to punish an extremely disruptive player. Thus an abusive act may become a sanction mechanism when it is turned against an offender. The same may happen in asynchronous systems, for example, when newsgroup users collectively agree to intentionally send spam-mail to disruptive people, which restricts use of their mailbox. These examples illustrate that disruptive behaviour used as punishment against abusive individuals may produce a powerful sanction mechanism.

\section{Conclusions and Outlook}

We have presented an analysis of existing behavioural rules and social conventions documented as netiquettes for both asynchronous (newsgroups, mailinglists, Email) and synchronous (chatrooms, MUDs) communication media. In real world groups and communities, social conventions provide a framework for the type of relationships and social atmosphere that they want to achieve. The aim of these conventions may be to provide an effective balance between the societal expectation of the community and the interests of 
individual participants. In our study of virtual environments, we found that netiquettes also seek to achieve this balance.

Although the Internet presents new possibilities for social entertainment and communication, many of the real world social regulations apply. However, the low level of awareness available makes learning the desirable behaviour through observation quite difficult. It is therefore important to learn it from reading the documented netiquettes. Netiquettes aim to facilitate group cohesion and the process of group formation. Following the netiquettes should help a community maintain and achieve its goals. Netiquettes often recommend the application of real life politeness. They contain some rules that are valid in general; some others are related to the technical medium, or to the specific purpose of the community. Many netiquettes address the multicultural dimension of the Internet. Rules protecting privacy, recognition of one's audience, and comments regarding polite behaviour reflect a constructive effort for an Internet environment that reflects an ideal real-world environment.

Major differences between synchronous and asynchronous media occur with respect to the kinds of disruptive behaviour that is to be prevented and the availability of sanction mechanisms. Prevention of flaming and spamming are of particular relevance in the netiquettes of asynchronous systems, i.e. general netiquettes, as well as in newsgroups and Email netiquettes. In chatrooms and MUDs, the creation of new identities may allow gender swapping and may result in social problems in the community. Nevertheless, maintaining anonymity is of high value in many of these communities.

Technical means for sanctions for offensive behaviour and for individual protection against known abusers are available in all media. There are more effective means available in synchronous media, i.e. chatrooms and MUDs as administrators are permanently available 
and may immediately intervene, delete offensive contributions and may even withdraw access to the service. These sanction mechanisms are often announced in the netiquettes.

To summarise, the documented netiquettes provided a narrative about Internet communities and about media specific differences. Our study provides evidence that the written down netiquettes aim to support the community building and maintenance process and to discourage media specific disruptive behaviour. We expect, with the growth of the Internet, the relevance of netiquettes will also increase as a means to support new users efforts to become respectable community members.

Acknowledgements: Special thanks to the anonymous reviewers for their feedback and to Konrad Klöckner who contributed to earlier parts of this study.

\section{References}

Astrian.net (2000). The Jargon Dictionary. http://info.astrian.net/jargon/terms/f/flame_war.html.

Barker, R.G. \& H.F. Wright (1955). Midwest and its Children. Reprint 1971. New York: Harper \& Row.

Bechar-Israeli, H. (1995). FROM $<$ Bonehead $>$ TO $<$ cLoNehEAd $>$ Nicknames, Play and Identity on Internet Relay CHAT $<1>$. Journal on Computer Mediated Communication, $1,2$. http://www.ascusc.org/jcmc/vol1/issue2/bechar.html.

Brotz, D.K. (1983). Message System Mores: Etiquette in Laurel. ACM Transactions on Office Information Systems, 1, 2, 179-192.

Bruckman, A.S. \& M. Resnik (1995). The MediaMOO Project: Constructionisms and Professional Community. Convergence, 1, 1, 94-109.

Ciskowski, D. \& C. Benedikt (1995). Don't be a TinyJerk! in MUDs: Exploring Virtual Worlds on the Internet. http://www.cwrl.utexas.edu/ claire/texts/netiquette.html.

Crott, H. (1979). Soziale Interaktion und Gruppenprozesse. Stuttgart: Kohlhammer.

Curtis, P. (1996). MUDding: Social Phenomena in Text-based Virtual Realities. In P. Ludlow (ed.): High Noon on the Electronic Frontier: Conceptual Issues in Cyberspace. Cambridge: MIT Press, 347-373.

Davis, F.D. \& J.J. Palladino (1995). Second Edition Psychology. Upper Saddle River, New Jersey: Prentice Hall.

Dery, M. (1994). Flame Wars. In M. Dery (ed.): Flame Wars - The Discourse of Cyberculture. Durham and London: Duke University Press, 1-10.

Dibbell, J. (1998). A Rape in Cyberspace. In R. Holeton (ed.): Composing Cyberspace: Identity, Community, and Knowledge in the Electronic Age. McGraw-Hill. http://www.mhhe.com/socscience/english/holeton/. 
Feldman, D., C. (1984). The Development and Enforcement of Group Norms. Academy of Management Review, 9, 1, 47-53.

Hamman, R., B. (1996). Cyborgasms - Cybersex amongst Multiple-Selves and Cyborgs in the NarrowBandwith Space of America Online Chat Rooms. MA. University of Essex. http:www.socio.demon.co.uk/Cyborgasm.html.

Hamman, R. (1997). History of the Internet, WWW, IRC, and the MUDs. http://www.socio.demon.co.uk/history.html.

Herrmann, F. (1995). Listserver Communication: The Discourse of Community Building. CSCL'95 Conference, Indianapolis. http://www-csc195.indiana.edu/csc195/herrmann.html.

Kehoe, B.P. (1992). Zen and the Art of the Internet: A Beginner's Guide. Upper Saddle River, NJ: Prentice Hall. http://www.cs.indiana.edu/docproject/zen/zen-1.0_toc.html\#SEC1.

Kelley, P. (1995). IRC World. http://159.148.118.3/personel/maris/RIGA/irc.htm.

Kollock, P. \& M. Smith (1994). Managing the Virtual Commons: Cooperation and Conflict in Computer Communities. In S. Herring (ed.): Computer-Mediated Communication: Linguistic, Social, and CrossCultural Perspectives. Amsterdam: John Benjamins, 109-128.

Lavato, S. (1997). MOO. http://whatis.com/moo.htm.

Lavato, S. (1998). MUD. http://whatis.com/mud.htm.

Mandel, T. \& G. van der Leun (1996). Rules of the Net. On-line Operating Instructions for Human Beings. New York: Hyperion.

Mann, L. (1972). Sozialpsychologie. Weinheim und Basel: BeltzVerlag.

Mitchell, W.J. (1996). City of Bits: Space, place, and the Infobahn. Cambridge: MIT Press.

Morgenes, P. (1997). Multi-User Dungeons. http://www.slac.com/morgenes/mud_def.html.

Oldenburg, R. (1989). The Great Good Place. New York: Paragon.

Pankoke-Babatz, U. (1984). Experiences with the KOMEX system as an inhouse CBMS. In H. T. Smith (ed.): Computer-based Message Services. Amsterdam: North-Holland, 193-200.

Pankoke-Babatz, U. (2000). Electronic Behavior Settings for CSCW. AI \& Society, 14, 1, 3-30.

Reid, E. (1991). Electropolis: Communication and Community on Internet Relay Chat. Honours Thesis. University of Melbourne. http://www.ee.mu.oz.au/papers/emr/electropolis.html.

Reid, E. (1994). Cultural Formations in Text-based Virtual Realities. University of Melbourne.

Reid, E. (1996). Text-based virtual realities: Identity and the cyborg body. In P. Ludlow (ed.): High Noon on the Electronic Frontier: Conceptual Issues in Cyberspace. Cambridge: MIT Press, 327-346.

Reid, E. (1999). Hierarchy and power. In M. Smith \& P. Kollock (eds.), Communities in Cyberspace. New York.: Routledge.

Rheingold, H. (1993). The Virtual Community. New York: Addison-Wesley.

Rheingold, H. (1996). A slice of my life in my virtual community. In P. Ludlow (ed.): Conceptual Issues in Cyberspace. Cambridge: MIT Press, 413-436.

Rospach, C.V. (1996). A Primer on How to Work With the Usenet Community. http://www.lib.ox.ac.uk/internet/news/faq/archive/usenet.primer.part1.html.

Short, J., E. Williams, \& C. Bruce (1976). The social psychology of telecommunications. London: John Wiley \&Sons, Ltd. 
Tannenbaum, S. (1996). Computer Networks. N.J.: Prentice-Hall.

Turkle, S. (1995). Life on Screen. New York: Simon \& Schuster.

Turkle, S. (1996). Virtuality and its discontents: Searching for community in cyberspace. The American Prospect, 24, 50-57.

\section{Referenced Netiquettes}

About.com (2000). Chatting online. http://chatting.about.com/internet/chatting/mpchat.htm.

America Online (1999). AOL Instant Messenger Web Chat Rules and Etiquette, http://www.aol.com/community/rules.html.

Bayer, J. (1998). Regeln für Usenet und Mailinglisten. http://asrv.asam.baynet.de/asaminfo/regeln.htm.

Chrystal Mush (1998). Introduction to Christal Mush. http://www.visi.com/ gyles19/CM/policy.manual.shtml.

CNN.com (1999). Discussion Messageboards. http://community.cnn.com.

Deeper Trouble (2000). Rules for MUD. http://sunsite.auc.dk:6860/rules.html.

Email Etiquette (1999). http://www.linfield.edu/policy/netiquette.html.

Excite (2000). People and Chat. http://www.excite.com/communities/resources/standards/chat.

HyperChat (2000). Welcome to HyperChat UK. http://hyperchat.co.uk/.

Letsgo (1997). Let's Go. http://www.letsgo.com.

MOO Connections (1999). http://web.nwe.ufl.edu/ tari/connections/.

Netiquette (1997). Rules of the Road. http://wwwlearning.berkeley.edu/Courses/AS128Sum97/day2/netiquette.html.

ParadisePlace (1999). Net for Newbies. http://www.paradiseplace.org.uk/other/usenet.htm.

Rainforest (2000). Chat-etiquette. http://www.geocities.com/RainForest/9900/netiq/netiq1.html.

RFC1855 (1995). Netiquette Guidelines. http://www.nccn.net/bultnbrd/faq/rfc1855.html.

WBS (1998). WBS. http://www.wbs.net.

Yahoo (2000). Terms of service for chatroom. http://docs.yahoo.com/info/terms.

YOYO (1997). Usenet and Netiquette. http://yoyo.cc.monash.edu.au/help/usenet.html. 


\section{Table 1: Percentage of netiquettes addressing desired behaviour}

\begin{tabular}{lcccccc}
\hline & $\begin{array}{c}\text { Awareness } \\
\text { of Audience }\end{array}$ & Privacy & Politeness & Style & Role Playing & $\begin{array}{c}\text { Total Desired } \\
\text { Behaviour }\end{array}$ \\
\hline General & $85 \%$ & $62 \%$ & $92 \%$ & $38 \%$ & - & $100 \%$ \\
Email & $71 \%$ & $71 \%$ & $86 \%$ & $86 \%$ & - & $93 \%$ \\
Newsgroups & $75 \%$ & $50 \%$ & $79 \%$ & $25 \%$ & - & $88 \%$ \\
Chatrooms & $56 \%$ & $50 \%$ & $33 \%$ & $33 \%$ & - & $83 \%$ \\
MUD & $71 \%$ & $47 \%$ & $76 \%$ & $12 \%$ & $24 \%$ & $94 \%$ \\
\hline
\end{tabular}

Table 2: Percentage of netiquettes containing rules concerning undesired behaviour

\begin{tabular}{lccccc}
\hline & $\begin{array}{c}\text { Undesired } \\
\text { Behaviour }\end{array}$ & Spamming & Flaming & $\begin{array}{c}\text { Sanction } \\
\text { Mechanisms }\end{array}$ & $\begin{array}{c}\text { Total } \\
\text { Undesired } \\
\text { Behaviour }\end{array}$ \\
\hline General & $54 \%$ & $46 \%$ & $69 \%$ & $8 \%$ & $77 \%$ \\
Email & $43 \%$ & $36 \%$ & $43 \%$ & $7 \%$ & $71 \%$ \\
Newsgroups & $38 \%$ & $38 \%$ & $63 \%$ & $21 \%$ & $83 \%$ \\
Chatrooms & $61 \%$ & $17 \%$ & $22 \%$ & $61 \%$ & $83 \%$ \\
MUD & $82 \%$ & $41 \%$ & $0 \%$ & $65 \%$ & $94 \%$ \\
\hline
\end{tabular}

